\title{
Revisiting Requirements Elicitation Techniques
}

\author{
Shreta Sharma \\ St. Xavier's College \\ JAIPUR - 302001, INDIA
}

\author{
S. K. Pandey \\ Board of Studies, The Institute of Chartered \\ Accountants of India (Set up by an Act of \\ Parliament),NOIDA - 201309, INDIA
}

\begin{abstract}
The importance of Requirements Engineering (RE) has been well recognized by the research community in the last decade. There is no doubt to say that requirements phase is the foundation of the entire Software Development Life Cycle (SDLC) on which the entire software is built. With the proper management of various activities falling under requirements phase, a project can deliver the right solution within the time and budget. Requirement elicitation, requirement specification, and requirement validation are the important stages to assure the quality of requirement documentations. Out of these, elicitation is the first major activity, which requires proper attention by Requirement Engineers and other related stakeholders. Literature reveals various elicitation techniques, which can be used for the purpose depending upon the nature of project/s. In this paper, an attempt is made to cover all the major elicitation techniques along with their significant aspects at one place. Such a review would enable the concerned stakeholders to understand and select the most appropriate technique to be used for their project/s.
\end{abstract}

\section{Keywords}

Software Engineering, Requirements Engineering, Requirements Elicitation, Requirement Elicitation Techniques.

\section{INTRODUCTION}

The foremost objective of Software Engineering (SE) practices and tools is to consistently produce high-quality software along with predictable budget restrictions and project schedules. Contrary to this, research surveys indicate that the majority of the software projects fail on these major issues such as quality, schedule and cost [1]. Researchers have identified significant causes behind the same and accordingly, they found that majority of the software fail due to poor requirements [1]. It is observed that various issues in software projects are mostly arising by a lack of clear understanding of the customers' requirements at the beginning of the project. These problems' nature and quality both continue to grow exponentially with the growth in software complexity and its versatility. The discipline of RE focuses on various activities related to requirements and tries to overcome these aforementioned issues right from the beginning in the SDLC [2]. RE is a branch of Software Engineering (SE), which refers to the process of formulating, documenting and maintaining software requirements [3]. The major aim of RE is to fulfill customer's needs and possibly exceeding them by using rigorous and well-defined methodologies. RE helps organizations to develop quality software systems within time and budget constraints offering true reflection of customer needs. There are various activities in RE viz. elicitation, analysis, specification and management etc.
Out of these activities, primary success factor of requirements elicitation is that requirements meet end user needs. However, practically, this outcome is difficult to achieve because users keep on changing their needs from time to time based on different aspects.

Requirements Elicitation is the process of discovering, extracting and gathering requirements for a system through communication with the stakeholders [3]. It has very high impact on subsequent design and development phases as well. Requirements elicitation is an ongoing problem area, which may cause even to the failure of the produced system. The process of requirements elicitation is generally accepted as one of the critical activities in the RE process. The main aim of this activity is to understand the stakeholders' needs and constraints, which are analyzed and specified with requirements. In reality, requirements elicitation is a multidimensional and iterative activity that heavily depends on the communication skills of requirements engineers and the commitment of the system stakeholders [2]. In literature, there are various techniques and methods for requirement elicitation activity, which provide a great support towards improving the overall quality of software.

In this paper, a comprehensive review of state-of-the-art techniques and practice of requirements elicitation has been accomplished by highlighting their key features. The main aim of this paper is to highlight all the major techniques, approaches, and methods used in requirements elicitation. Beyond this 'Introduction' on the background details, rest of the paper is organized as follows: Section II presents a brief overview of 'RE Process' whereas various elicitation techniques have been given in Section III. Finally, 'Conclusion and Future Work' are reported in Section IV.

\section{RE PROCESS}

$\mathrm{RE}$ is one of the most important areas of software development. During this stage, the customer and Requirement Engineers come to an agreement as to "what constitutes the software to be developed'. This is a critical stage because anything that is (or is not) resolved at this time, will be carried down through the rest of the software development lifecycle. Good RE process is therefore essential for successful system development [4]. RE process have several phases, which facilitate to understand the customers' needs, define the system requirements and constraints, analyze them, evaluate their feasibility, determine customers' real need, validate requirements specification and manage the requirements. Basically, there are five major phases of RE, which are given as follows [5]: 


\subsection{Elicitation}

It is regarded as the first phase of the RE process and normally considered as the process of finding out 'what are the real needs of the customers as well as of the system' [5]. It also includes activities to explore 'how the software can meet the stakeholders' goals' and 'what alternatives might exist' [6].

\subsection{Analysis and Negotiation}

It is the second phase, which consists of a set of activities aimed to discover problems within the system requirements and achieve agreement on changes to satisfy all system stakeholders. If an analyst discovers some problems with the requirements during the analysis phase, such requirements are referred back to the elicitation phase. This process is related to the requirements that are incomplete, ambiguous and/or conflicting. Negotiation part is known as 'the process of discussing conflicts in requirements and finding some compromise which all of the stakeholders can live with' [5]. The principle of this process should be objective, where the judgments and the compromise for the system requirements should be based on technical and organizational needs. All the conflict requirements identified during the analysis process should be negotiated and discussed individually with the stakeholders in order to resolve the conflicts $[5,7]$.

\subsection{Documentation}

This is the third phase of RE process. Once the requirements have been analyzed, it is important to record them in order to make them formal through proper specification mechanism. During this phase, the team organizes the requirements in such a way that ascertains their clarity, consistency, and traceability etc. This phase is extremely important because often 'the document produced during specification is what the rest of the development stages will be based upon'.

\subsection{Validation}

This fourth phase ensures that models and documentation accurately express the stakeholders' needs along with checking the final draft of requirements document for conflicts, omissions and deviations from different standards.

\subsection{Management}

This is the fifth and last phase of requirements lifecycle. It is similar to the maintenance of a software system. Some of the most important maintenance tasks during this phase include the updating of the requirements as well as the degree of evolution support that the approach provides.

\section{REQUIREMENT ELICITATION TECHNIQUES}

Requirements elicitation is a complex process involving many activities with a variety of available techniques, approaches for performing them. Several researches and practices within RE have been largely directed towards improving the elicitation process through development of various techniques. A variety of trend setting elicitation techniques used by various RE practitioners are hereby given under various heads:

\subsection{Traditional Techniques}

As projects became more and more complex, the needs to organize the development work also arose. The 'waterfall' process was adapted, and development work was thought of a process; when one phase is concluded, the next one begins in a linear timeline with the waterfall. The early elicitation methods are now-a-days called traditional techniques. These include a broad class of generic data-gathering techniques such as questionnaires and surveys, interviews, and analysis of existing documentation such as organizational charts, process models or standards, and user or other manuals of existing systems. These are given as follows:

\subsubsection{Interviews}

A typical conversational traditional method is interviews. It is a commonly used technique in requirements elicitation because it is essentially human based social activity [8]. It is an efficient approach to gather large size of information; rapidly and resultant useful information can significantly depend on the skill of the interviewer. Interviewing is not simply a matter of asking questions; it requires development of some general social skills, the ability to listen, and knowledge of a variety of interviewing policies. A qualitative Interview is basically produced by an experienced analyst, who has some fundamental knowledge about the application domain [9].Generally interviews are divided into two groups: structured and unstructured, which are discussed as follows:

- Structure: Structured interviews are grounded on predetermined fixed set of questions along with detailed information. It enables the Requirement Engineers to examine the level of understanding a respondent has about a particular topic. The quality and usefulness of the information is highly dependent upon the quality of the questions asked [10]. Templates are also a part of this procedure that provides an easier way to requirements elicitation. While structured interviews tend to limit the exploration of new ideas and expressions, they are generally measured to be demanding and effective [8].

- Unstructured: The unstructured interview technique was established in the disciplines of anthropology and sociology as a technique to elicit people's social actualities. Unstructured interviews are most useful when you want to focus on deep understanding of a particular portent within a particular social environment [11]. Unstructured interview are conversational in nature where do not need to prepare any predefine questions, and the information from the stakeholders is in open discussions. But sometime there is the risk that some topics may be completely neglected and some topic gain focus in depth on some areas and not enough in others.

\subsubsection{Surveys/Questionnaires}

Questionnaires are mostly used as a simple tool, which may consist of open and/or closed questions during the initial phase of requirements elicitation. These are one of the methods of gathering requirements in less cost and time. This is an efficient way to collect information from multiple stakeholders quickly with avoiding the large amounts of redundant and irrelevant data. Questionnaires must be clear, defined and concise along with the domain knowledge [12]. Questionnaires provide no mechanism for the participants to request clarification or correct misunderstandings. 


\subsubsection{Introspection}

The introspection technique is the base point for other requirement elicitation techniques. Introspection is the idea of the RE about the wish and needs of the stakeholders for the systems. Introspection is only really effective when the analyst is not only expert in the domain knowledge and goals of the system but also having knowledge in the business processes performed by the users [7].

\subsubsection{Reading Existing Documents}

Document means manuals, input forms, output forms, diagrams of how the current system works, organization charts showing hierarchy of users and manager responsibilities, job descriptions for the people, who work with the current system, procedure manuals, program codes for the applications associated with the current system, etc. [4]. Documents are a very good source of information about user needs and the current system.

\subsubsection{Meetings}

Requirements can be well elicited through formal or informal meetings. This technique is especially useful in case of a conflict among different stakeholders [4].

\subsection{Collaborative Techniques}

All techniques are good and efficient in some certain perspective and environments. It is often a good approach to collaborating different elicitation techniques at one place for developing requirements. The collaboration of various techniques helps to experts in discovers the basic concept and improves the generic knowledge about application domain. Rather than combining separate methods, the collaborative technique procedure constructs a whole procedure by methodically merging traditional, contextual, and cognitive into single technique. Experts and stakeholder representatives communicate and coordinate in different ways to extent a mutual understanding of the desired product [8]. Collaborating techniques includes brainstorming, prototyping, JAD/RAD sessions etc., which are given as follows:

\subsubsection{Brainstorming}

Brainstorming is a group technique for generating preliminary ideas about the software to be developed in the project. It is a process where participants from different stakeholder groups take part in familiar discussion to quickly produce as many ideas as possible without focusing on any one in individual. This technique is often used to develop the preliminary major statement for the project and target final software. This brainstorming technique has two phases that is Generation Phase, which offer as many ideas without discussing merits of the ideas and other one is consolidations, which discussed the ideas, revised and organized. One of the major profit in using brainstorming is that it inspires wide ideas and expression, and includes the discovery of new and advanced procedures to existing problems [7].

\subsubsection{JAD/RAD Sessions}

Joint Application Development (JAD) and Rapid Application Development (RAD) accentuate user involvement through group meetings with balanced facilitator. A major difference between $\mathrm{JAD}$ and brainstorming is that JAD sessions are well defined steps including actions and roles of participant, another is the stakeholders and the users are also allowed to participate and discuss on the design of the proposed system expect this whole process is similar to brainstorming. The major goal of the system has already been recognized before the participation of stakeholders. The communication between stakeholders and the users remain active until the final requirements are collected [9].

\subsubsection{Prototyping}

Prototyping is an efficient two-way technique based on close interaction between the experts and the end-user for getting feedback from users. Prototypes are typically developed using preliminary requirements or existing examples of similar systems [8]. This technique is particularly useful when developing human-computer interfaces, or where the stakeholders are unacquainted with the available solutions [12]. Other elicitation techniques such as JAD and interviews are used with the combination of prototype.

\subsection{Contextual Techniques}

Contextual Technique is a combination unstructured interview, workplace opinion, and prototyping. The goal of this technique is to collect comprehensive data about stakeholders, their working procedures, environments, patterns, flow and other relevant area regarding their daily work and then to interpret the data to gain in-depth understanding of requirements and suitable system design. This method is used for interactive systems design where user interface design is critical [13]. Various techniques under this category are given as follows:

\subsubsection{Ethnographic Techniques}

Ethnography is a social science elicitation technique that supports context-aware technique requirements. This technique is developed by anthropologists for understanding people within their social and cultural perceptions. The fundamental basis of ethnography is that people's actions and thoughts are dependent on a vast range of factors [14]. These techniques are especially used when delivering contextual issues such as usability and exploring collaborative work locations [8].

\subsubsection{Discourse Analysis}

This is an extended version of ethnography techniques. It consists of two sub techniques called Conversation Analysis and Speech Act Analysis. Discourse analysis offers various tools to examine systematically the fundamental substance of requirements elicitation i.e. interactional talk. This analysis employs sociolinguistic methods for specifying the linguistic features of different types of discourse units and the way they are tied together to create meaning, with critically examining social context [15].

\subsubsection{Sociotechnical Methods (Soft Systems Analysis)}

These produce informal models of a socio-technical system. The primary consideration in these techniques is the system, people and the organization. Rather, they are ways of understanding a problem and its organizational context. Software Systems Methodology (SSM) is probably the best known of these methods. The essence of SSM is its recognition that systems are embedded in a wider human and organizational context [16] [17]. 


\subsection{Cognitive Techniques}

These elicitation techniques are based on multidisciplinary approaches as the information on knowledge elicitation methods is widely scattered across the fields of Psychology, Business Management, Education, Counseling, Cognitive Science, Linguistics, Philosophy, Knowledge Engineering and Anthropology [18]. This section provides an overview of knowledge elicitation techniques along with some other useful techniques, which are given as follows:

\subsubsection{Protocol Analysis}

This technique is based on the protocols created from vocalizing behavior either 'think-aloud' or retrospective. The idea is to analyze protocols to reveal requirements. The major advantages are direct verbalization of cognitive activities, embedded in the work context. Further, this technique is found to be good at revealing interaction problems with existing systems. However, there are some disadvantages also as interpretation requires introspection; hence it is unreliable and there is no social dimension [16].

\subsubsection{Knowledge Acquisition Techniques}

There are various knowledge acquisition techniques available in the literature. Some of them are given as follows:

- Card Sorting: Card Sorting is an established method for requirement elicitation. It has been established that card numbering has many affirmative phases that makes it efficient tool for requirement elicitation. Card sorting requires sorting a series of domain entities card from stockholders [19].

- Repertory Grids: It is a simple knowledge elicitation technique normally used by clinical psychologists. After identifying a small set of elements e.g. objects, entities, the user is asked to define some constructs like attributes, slots, which characterize those elements. In addition, construct values can be given for each element on a limited scale between two range end points (the left and right poles). This is essentially qualitative information, which can be elicited through the repertory grid tool. The method also helps to elicit an initial representation, which can afterwards be refined by applying other established refinement mechanisms [20].

- Proximity Scaling Techniques: In this technique, for some given domain objects, a set of dimensions are derived for classifying them. The first step is pairwise proximity assessment among domain elements, which captures tacit knowledge of expert. Afterwards, automated statistical analysis is done to build multi-dimensional space to classify the objects. Major advantages are that it helps to elicit mental models where complex multivariate data is concerned and it is good for eliciting tacit knowledge also. However, it requires an agreed-upon set of objects and only models classification knowledge, not performance knowledge [16].

- Laddering: This is a structured questioning technique derived from the repertory grid technique, enabling a hierarchy of concepts to be established.
Laddering is best fit for integration with a protocol editor in which the output of an editor is used as a conceptual input. These tools may be used early in the knowledge acquisition process by forming/refining initial knowledge hierarchies [21].

\subsubsection{Task Analysis}

This technique uses a variety of interview and observation strategies to capture a description of the knowledge that experts use to perform complex tasks. Here, complex tasks are those where performance requires the integrated use of both controlled (conscious, conceptual) and automated (unconscious, procedural or strategic) knowledge to perform tasks [22].

\subsection{Innovative Techniques}

In spite of numerous elicitation techniques available in RE, there are still several unresolved issues, which requires further attention by the research community. It is expected that some other elicitation techniques may be evolved in future offering solution/s to these issues. Accordingly, an innovative category of elicitation techniques has been proposed under which all the newly proposed techniques will be put forth[23].

One of such innovative techniques is Throwaway Paper Prototype. A paper prototype is a visual representation of 'what the System will look like'. Normally, users are expected to draw the features through a pen/pencil on paper and share with Requirement Engineers. Alternatively, a graphics program can also be used for the purpose. Although, in most of the cases, paper prototype is used as a part of the usability testing, where the user gets a feel of the User Interface but by applying the same technique in requirements elicitation, satisfactory results have been obtained. These tryout results indicate that the paper prototype method for requirements elicitation is a suitable method for small and medium sized projects. Other such techniques may also be placed in this category in future.

\section{CONCLUSION AND FUTURE WORK}

The paper presented an overview of the complete RE process by discussing all the major activities starting from requirements elicitation till their management. The paper also offered a brief but complete description of various elicitation techniques used by RE practitioners worldwide. Earnest efforts have been made to place all the major elicitation techniques in the paper. In addition, a provision has also been made for suitable placement of the elicitation techniques to be proposed in future by the researchers in the related area.

Future work may be to conduct a comparative study of these elicitation techniques based on some well-defined parameters. Another work may be to identify the current concerns and future prospects with reference to requirements elicitation. Afterward, novel ideas/approaches may be suggested to overcome these concerns along with strong validation results. This work may provide a significant guidance to the $\mathrm{RE}$ practitioners for developing a quality software.

\section{REFERENCES}

[1] Pandey, S. K. and Mustafa, K. 2010. Risk Assessment Framework (RAF). International Journal of Advanced Research in Computer Science. Vol. 01. pp. 423-432. 
[2] Pandey, S. K. and Batra, Mona 2013. Formal Methods in Requirements Phase of SDLC. International Journal of Computer Applications. Published by Foundation of Computer Science. New York. USA.pp.7-14

[3] Pandey, S. K. and Mustafa, K. 2010. Recent Advances in SRE Research. International Journal of Computer Science and Engineering. Vol. 02. pp. 1079-1085.

[4] Mead, N. 1996. Security Requirements Engineering. Carnegie Mellon University. Last Retrieved March 20, 2008. https://buildsecurityin.us-cert.gov/daisy/bsi/ articles/bestpractices/requirements/243.html

[5] Kotonya, G. and Sommerville I. 1998. Requirements Engineering Processes and Techniques, John Wiley \& Sons Ltd, England.

[6] Hofmann, H.F. and Lehner F. 2001. Requirements Engineering As a Success Factor In Software Projects. IEEE Software. IEEE. 18(4). pp. 58-66.

[7] Paetsch, F., Eberlein, A. and Maurer, F. 2003. Requirements Engineering and Agile Software Development. Enabling Technologies, IEEE. pp. 308-313.

[8] Didar, Zowghi and Chad, Coulin 2005. Requirements Elicitation: A Survey of Techniques, Approaches and Tools. Engineering and Managing Software Requirements. pp.19-46.

[9] Chen, Ying 2012. Requirement Elicitation Techniques. http://www.umsl.edu/ ycnx6/. Last Retrieved on July 22, 2013.

[10] http://www.sociology.org.uk/methsi.pdf. Last Retrieved on July 10, 2013.

[11] Yan Zhang and Barbara M. Wildemuth 2009. Unstructured Interviews,1-9.pp.1-9. Last Retrieved on July20, 2013. https://www.ischool.utexas.edu/ yanz/Unstructured_intervi ews.pdf

[12] Shams-Ul-Arif, Khan, Qadeem and Gahyyur, S. A. K. 2009. Requirements Engineering Processes, Tools/Technologies, \& Methodologies. International Journal of Reviews in Computing. pp.42-56.

[13] Jarkko Viinamäki 2004. Requirements Elicitation: Contextual Inquiry. T-76.652: Seminar on User and Customer Requirements Engineering. pp. 1-10.

[14] Social Innovator: Ethnography research techniques, Last Retrieved on July 20, 2013 http://www.socialinn ovator.info/ process-social-innovation/prompts-andinspirations/research-and-mapping/ethnographic-researchtechniques

[15] Rosio Alvarez 2002. Confessions of an information worker: a critical analysis of information requirements discourse. Inform. Org. pp. 85 -107.

[16] http://www.cs.toronto.edu/ sme/CSC2106S/slides /04elicitation- techniques.pdf, Last Retrieved on July 13, 2013.

[17] Volha Bryl, Paolo Giorgini and John Mylopoulos 2009. Designing Socio-Technical Systems: From Stakeholder
Goals to Social Networks. Requirements Engineering 14(1), pp.47-70.

[18]Cooke, N. J. 1994. Varieties of knowledge elicitation techniques. International Journal of Human-Computer Studies.pp.2-59

[19] Nurmuliani, Nur. Zowghi, Didar and Susan P. Williams 2009. Using Card Sorting Technique to Classify Requirements Change

[20] http://www.hf.faa.gov/workbenchtools /default. aspx $?$ rPage $=$ Tooldetails $\&$ subCatId $=25 \&$ toolID $=228$, Last Retrieved on July 10, 2013.

[21] Corbridge, C., Rugg, G., Major, N.P., Shadbolt, N.R. and Burton, A.M. 1994. Laddering: technique and tool use in knowledge acquisition. Knowledge Acquisition 6.pp.315341.

[22]http://www.cogtech.usc.edu/publications/clark_etal_ognitiv e_task_analysis_chapter.pdf, Last Retrieved on July 10, 2013. International Requirements Engineering Conference

[23] Vijayan, Jaya and Raju, G. 2011. A New approach to Requirements Elicitation Using Paper Prototype. International Journal of Advanced Science and Technology.pp.9-16

\section{AUTHORS}

Shreta Sharma is currently working as a Faculty in the Department of Computer Science, St. Xavier's College, Jaipur. Prior to this, she was associated with Natural Software Pvt. Ltd., Jaipur as a Software Developer. She has an excellent academic background right from the school level. Under the InstituteIndustry linkage program, she delivers expert lectures on various area of Computer Science. She has contributed many research papers in the conferences of national repute. Her area of research includes Artificial Intelligence, Requirements Engineering, E-learning and Software Security.

Dr. Santosh K. Pandey is presently working as a Faculty of Information Technology with Board of Studies, The Institute of Chartered Accountants of India (Set up by an Act of Parliament) New Delhi. Prior to this, he worked with the Department of Computer Science, Jamia Millia Islamia (A Central University) New Delhi and Directorate of Education, Govt. of NCT of Delhi. He has a rich Academics \& Research experience in various areas of Computer Science. His research interest includes: Software Security, Requirements Engineering, Security Policies and Standards, Formal Methods, Cloud Computing, Security Metrics, Vulnerability Assessment etc. He has published around 46 high quality research papers and articles in various acclaimed International/National Journals (including IEEE, ACM, CSI) and Proceedings of the reputed International/ National Conferences (including Springer). Out of these publications, most of them have good citation records. He has been nominated in the board of editors/reviewers of various peer-reviewed and refereed Journals. In addition, he has also served as a Program Committee Member of several reputed conferences in India as well as abroad. He has also been designated in various academic/research committees by the government organizations as well as software companies as a subject expert. 